\title{
Diffusion of a polymer in a random medium
}

\author{
G. C. Martinez-Mekler (*) and M. A. Moore \\ Department of Theoretical Physics, The University, Manchester, M13 9PL, U.K.
}

(Reçu le 29 juin 1981, accepté le 28 juillet 1981)

\begin{abstract}
Résumé. - Il est montré que l'effet d'un potentiel aléatoire sur le coefficient de diffusion d'un polymère correspondant à une perturbation au sens du groupe de renormalisation pour des dimensions spatiales inférieures à quatre. Des tentatives « d'amélioration » du développement en perturbation à l'aide du groupe de renormalisation n'aboutissent pas, faute de pouvoir localiser un point fixe accessible. Des hypothèses sur les implications physiques de ces résultats sont données.
\end{abstract}

\begin{abstract}
The effect of a random potential on the diffusion coefficient of a polymer is shown to be a relevant perturbation in the renormalization group sense below four dimensions. Attempts to " improve " the perturbation expansion by means of the renormalization group failed as it was not possible to locate a stable accessible fixed point. Speculations on the physical implications of these results are given.
\end{abstract}

In this letter we examine the statics and dynamics of a flexible linear polymer moving in a fixed (quenched) random potential. This potential might be envisaged as being produced by hard spheres placed at random throughout space whose diameters are small compared to the length of the polymer molecule. Such a model might serve as a crude approximation for a polymer diffusing through a medium of closely packed small particles, e.g. oil through sand. However, our chief motivation in studying this problem was to try to understand what de Gennes refers to as « one of the major unsolved problems of polymer physics " [1]. This is the discrepancy between the reptation model prediction for the molecular weight dependence of the viscosity $\eta$ of a melt and experiment. Experimentally it is found that $\eta \sim N^{m_{\eta}}$ where $m_{\eta}$ is of order 3.3 to 3.4 while the reptation model prediction for $m_{\eta}$ is 3 .

The reptation model is usually introduced by first considering a system which is much simpler than a polymer melt but which still shows nontrivial entanglement effects. This is a single chain trapped in a fixed network [1]. The chain has to move through the network in a snake-like fashion called "reptation". The wriggling of a polymer in two dimensions amongst fixed randomly placed discs is also of this character. In the case we want to understand, viz, polymer

(*) Now at Instituto de Fisica, UNAM, Apartado Postal 20-364, Mexico 20, D.F., Mexico. motion in a random potential in three dimensions, the randomly placed spheres, do not provide topological constraints on the motion of the chain. We hope, nevertheless, that the motion falls into the same " universality class " as that through a fixed network. The presence of topological constraints makes the network problem intractable. However, the random potential problem is more amenable to standard techniques, such as those provided by the renormalization group.

The bead-spring Rouse model [2] will be adopted to describe the dynamics of the polymer. The equations of motion are

$$
\dot{R}_{j \alpha}=\mu\left[-\frac{\partial U}{\partial R_{j \alpha}}+f_{j \alpha}(t)\right]
$$

where $\mathbf{R}_{j}(t)$ is the position vector of the $j$ th bead $(j=1,2, \ldots, N)$ and $\alpha=1, \ldots, d$ labels its Cartesian components. The dimensionality of the system is $d$. $\mu$ is the friction coefficient and we have supposed that the presence of the random potential screens out the long-range hydrodynamic interactions. The effects of random collisions with solvent molecules is represented by the random force $\mathbf{f}_{j}(t)$ whose distribution is taken as Gaussian. The potential energy $\left(k_{\mathrm{B}} T\right)^{-1}$

$$
U\{R\}=U_{0}\{R\}+U_{1}\{R\}+U_{2}\{R\}
$$

where $U_{0}=\left(2 b^{2}\right)^{-1} \sum_{j=1}^{N-1}\left|\mathbf{R}_{j+1}-\mathbf{R}_{j}\right|^{2} \quad$ represents 
harmonic forces between neighbouring beads ; $U_{1}\{R\}$ is the potential energy of interaction between them, $U_{1}=2^{-1} \sum_{i, j} V\left(\mathbf{R}_{i}-\mathbf{R}_{j}\right)$ where $V\left(\mathbf{R}_{i}-\mathbf{R}_{j}\right)$ is the excluded volume interaction between the beads $i$ and $j$ and will be taken to be $V \delta\left(\mathbf{R}_{i}-\mathbf{R}_{j}\right) . U_{2}\{R\}$ is the potential energy of the beads in the random potential; $U_{2}=\sum_{j} \varphi\left(\mathbf{R}_{j}\right)$.

The time evolution of the polymer configuration distribution function $\rho[\{R\} ; t]$ is governed by the Liouville operator

$$
A=\sum_{j, \alpha}\left(k_{\mathrm{B}} T \frac{\partial}{\partial R_{j \alpha}}-\frac{\partial U}{\partial R_{j \alpha}}\right) \mu \frac{\partial}{\partial R_{j \alpha}}
$$

according to

$$
\dot{\rho}=A \rho, \quad P=Z^{-1} \exp \left(-U\{R\} / k_{\mathrm{B}} T\right) \rho
$$

where $Z=\int \mathrm{d}\{R\} \exp \left(-U\{R\} / k_{\mathrm{B}} T\right)$ is the ordinary partition function [3]. The characteristic relaxation times of the system are the inverse of the eigenvalues $\lambda_{n}$,

$$
A v_{n}\{R\}=-\lambda_{n} v_{n}\{R\} .
$$

If the excluded volume and the random potential are neglected, $A \rightarrow A^{(0)}$ and the equations (1)-(4) are directly soluble in terms of the normal (Rouse) coordinates [3]. The centre of mass motion separates out completely allowing one to label the eigenstates $\quad v_{n \mathbf{q}}^{(0)}=v_{n}^{(0)} \exp \left[i \mathbf{q} \cdot\left(\sum_{l} \mathbf{R}_{l}\right) / N\right] \equiv|n \mathbf{q}\rangle$, where $n=0,1,2, \ldots$, labels the eigenstates of the internal relaxation processes and $\mathbf{q}$ is the wave-vector of the centre of mass. Likewise $\lambda_{n q}^{(0)}=\lambda_{n}^{(0)}+\gamma q^{2} / N$ which implies that the dynamics of the centre of mass corresponds to the diffusion of a particle with diffusion coefficient $D=\gamma / N$, where $\gamma=\mu k_{\mathrm{B}} T$. Note $v_{0}^{(0)}=1$ and $\lambda_{0}^{(0)}=0$. There results are also recovered for any $U\{R\}=U\left(\mathbf{R}_{i}-\mathbf{R}_{j}\right)$ as can be seen by summing equation (1) over all beads. However, the random potential makes $U\{R\}$ non-translationally invariant and this alters the diffusion coefficient. The reptation model predicts that the molecular weight dependence of $D$ changes to $N^{-2}$ [1]. The aim of this letter is to try to find how $D$ is affected by the random potential. Our technique is perturbation expansions in $U_{2}\{R\}$.

It is first necessary to specify the random potential $\varphi(\mathbf{R})$. We shall take it to be Gaussian :

$$
\overline{\varphi(\mathbf{R})}=0, \quad \overline{\varphi(\mathbf{R}) \varphi\left(\mathbf{R}^{\prime}\right)}=\Delta \delta\left(\mathbf{R}-\mathbf{R}^{\prime}\right) .
$$

A hard-sphere potential involves higher cumulants which turn out to be irrelevant near four dimensions (as happens in a similar context in reference [4]). A non-vanishing first cumulant could be considered but inspection of equation (2) shows that any constant added to $U\{R\}$ drops out of the Liouville operator $A$. If we write $\varphi(\mathbf{R})$ in its Fourier representation, the contribution $A^{(2)}$ of the random potential to $A$ can then be expressed as

$$
A^{(2)}=-i \gamma \int \frac{\mathrm{d} \mathbf{p}}{(2 \pi)^{d}} \varphi_{p} \sum_{l, \alpha} \exp \left(i \mathbf{p} . \mathbf{R}_{l}\right) p_{\alpha} \frac{\partial}{\partial R_{l \alpha}}
$$

Let $\delta \lambda_{k}$ denote the change in the eigenvalue $\lambda_{0 \mathbf{k}}^{(0)}$ due to the random potential. To second order in a perturbation expansion in $A^{(2)}$

$$
\delta \lambda_{k}=\left\langle 0 \mathbf{k}\left|A^{(2)}\right| 0 \mathbf{k}\right\rangle-\sum_{n \mathbf{q}}^{1}\left\langle 0 \mathbf{k}\left|A^{(2)}\right| n \mathbf{q}\right\rangle\left\langle n \mathbf{q}\left|A^{(2)}\right| 0 \mathbf{k}\right\rangle /\left(\lambda_{0 \mathbf{k}}^{(0)}-\lambda_{n \mathbf{q}}^{(0)}\right) .
$$

$\left\langle n \mathbf{q}\left|A^{(2)}\right| 0 \mathbf{k}\right\rangle$ is shorthand for

$$
Z_{0}^{-1} \int \mathrm{d}\{R\} \exp \left(-U_{0}\{R\} / k_{\mathrm{B}} T\right) v_{n}^{(0)} \exp -i \mathbf{q} \cdot\left(\sum_{j} \mathbf{R}_{j}\right) / N A^{(2)} \exp i \mathbf{k} \cdot\left(\sum_{l} \mathbf{R}_{l}\right) / N
$$

On averaging $\delta \lambda_{k}$ over the random potential the first term vanishes as $\bar{\varphi}_{\mathbf{p}}=0$ while the second term gives

$$
\begin{aligned}
& \delta \lambda_{\mathbf{k}}=-\Delta \gamma^{2} \int \frac{\mathrm{d} \mathbf{p}}{(2 \pi)^{d}} \sum_{n \mathbf{q}}^{1}\left\langle 0 \mathbf{k}\left|\sum_{l, \alpha} \exp \left(-i \mathbf{p} . \mathbf{R}_{l}\right) p_{\alpha} \frac{\partial}{\partial R_{l \alpha}}\right| n \mathbf{q}\right\rangle \times \\
& \times\left\langle n \mathbf{q}\left|\sum_{j \beta} \exp \left(i \mathbf{p} . \mathbf{R}_{j}\right) p_{\beta} \frac{\partial}{\partial R_{j \beta}}\right| 0 \mathbf{k}\right\rangle /\left(\lambda_{0 \mathbf{k}}^{(0)}-\lambda_{n \mathbf{q}}^{(0)}\right),
\end{aligned}
$$

after using $\overline{\varphi_{\mathbf{p}} \varphi_{\mathbf{p}^{\prime}}}=\Delta \delta\left(\mathbf{p}+\mathbf{p}^{\prime}\right)$. The second matrix element in equation (8) equals

$$
i(\mathbf{k} \cdot \mathbf{p})\left\langle n \mathbf{q}\left|\sum_{j} \exp i \mathbf{p} . \mathbf{R}_{j}\right| 0 \mathbf{k}\right\rangle / N \text {. }
$$


If one were to integrate out the centre of mass coordinate in this expression one would find that it vanishes unless $\mathbf{q}=\mathbf{p}+\mathbf{k}$. The « energy » denominator can then be expressed for small $\mathbf{k}$ as

$$
\begin{aligned}
\left(\lambda_{0 \mathbf{k}}^{(0)}-\lambda_{n \mathbf{p}+\mathbf{k}}^{(0)}\right)^{-1} & \approx-\left(\lambda_{m \mathbf{p}}^{(0)}\right)^{-1}+2 \gamma(\mathbf{k} \cdot \mathbf{p}) /\left(N \lambda_{\mathbf{m p}}^{(0) 2}\right)+\cdots \\
& =\int_{0}^{\infty} \mathrm{d} t(-1+2 \gamma(\mathbf{k} \cdot \mathbf{p}) t / N) \exp -t \lambda_{\mathrm{np}}^{(0)}
\end{aligned}
$$

But for any two functions $G\{R(0)\}$ and $F\{R(t)\}$

$$
\begin{aligned}
\langle m|G\{R(0)\} F\{R(t)\}| m\rangle & =\langle m|G\{R\} \exp A t F\{R\}| m\rangle \\
& =\sum_{n}\langle m|G\{R\}| n\rangle\langle n|F\{R\}| m\rangle \exp -\lambda_{n} t .
\end{aligned}
$$

Hence

$$
\begin{aligned}
\delta \lambda_{\mathbf{k}}=-i \Delta \gamma^{2}(\mathbf{k} \cdot \mathbf{p}) / N \int \frac{\mathrm{d} \mathbf{p}}{(2 \pi)^{d}} \int_{0}^{\infty} \mathrm{d} t(-1+2 \gamma(\mathbf{k} \cdot \mathbf{p}) t / N) \times \\
\times\langle 0 \mathbf{k}| \sum_{l, \alpha} \exp \left(-i \mathbf{p} \cdot \mathbf{R}_{l}(0)\right) p_{\alpha} \frac{\partial}{\partial R_{l \alpha}(0)}\left\{\sum_{j} \exp i \mathbf{p} \cdot \mathbf{R}_{j}(t)|0 \mathbf{k}\rangle\right\} .
\end{aligned}
$$

Carrying out the differentiation with respect to $R_{l \alpha}(0)$ of the terms in the curly brackets in equation (9) produces two terms:

$$
\sum_{j} \exp \left(i \mathbf{p} \cdot \mathbf{R}_{j}(t)\right)\left[i p_{\alpha} G_{j l}(t)+i k_{\alpha} / N\right]|0 \mathbf{k}\rangle
$$

where the identity $\delta R_{j \beta}(t) / \delta R_{l \alpha}(0)=\delta_{\alpha \beta} G_{j l}(t)$ has been used. $G_{j l}(t)$ is the propagator for equation (1) for $U_{1}=0=U_{2}$ and is given by

$$
\begin{aligned}
G_{j l}(t)=(2 \pi)^{-1} & \int_{-\pi}^{\pi} \mathrm{d} y \times \\
& \times \exp \left(i y(j-l)-2 \gamma(1-\cos y) t / b^{2}\right)
\end{aligned}
$$

as $N \rightarrow \infty$ [5]. Then in the limit $k \rightarrow 0$

$$
\begin{aligned}
\delta \lambda_{k}=\Delta(\gamma / N)^{2} & \int \frac{\mathrm{d} \mathbf{p}}{(2 \pi)^{d}}(\mathbf{k} \cdot \mathbf{p})^{2} \int_{0}^{\infty} \mathrm{d} t \times \\
& \sum_{l, j} S_{l, j}(\mathbf{p}, t)\left[1-2 \gamma p^{2} t G_{l, j}(t)\right]
\end{aligned}
$$

where

$S_{l, j}(\mathbf{p}, t)=\left\langle 00\left|\exp \left(-i \mathbf{p} \cdot \mathbf{R}_{l}(0)+i \mathbf{p} . \mathbf{R}_{j}(t)\right)\right| 00\right\rangle$

for at this stage $|0 \mathbf{k}\rangle$ can be replaced by $|00\rangle$. From [5]

$S_{l, j}(\mathbf{p}, t)=\exp -\frac{p^{2} b^{2}}{2} \times$

$$
\times\left[s+2\left(\frac{\gamma t}{\pi b^{2}}\right)^{1 / 2} g\left(b^{2} s^{2} / 4 \gamma t\right)\right],
$$

with

$$
g(u)=\int_{1}^{\infty} \mathrm{d} y y^{-1} \exp -u y^{2} \text { and } S=|l-j|
$$

Eq. (11) is valid for $\gamma t \gg b^{2}$ and $N^{-1 / 2} \ll p b \ll 1$. Replacing $\sum_{l, j}$ in (10) by $N \int \mathrm{d} s$, equation (10) can be reduced to

$$
\delta \lambda_{k}=16 \frac{\gamma \Delta}{N b^{4}}\left[\frac{6}{(4 \pi)^{1 / 2}} C_{1}-C_{2}\right] \int \frac{\mathrm{d} \mathbf{p}}{(2 \pi)^{d}} \frac{(\mathbf{k} \cdot \mathbf{p})^{2}}{p^{6}}
$$

where

$$
\begin{aligned}
C_{1} & =\int_{0}^{\infty} \mathrm{d} \lambda \exp \left(-\lambda^{2}\right) /\left[\lambda+\pi^{-1 / 2} g\left(\lambda^{2}\right)\right]^{4} \\
& =2.46557 \\
C_{2} & =\int_{0}^{\infty} \mathrm{d} \lambda \exp \left(-\lambda^{2}\right) /\left[\lambda+\pi^{-1 / 2} g\left(\lambda^{2}\right)\right]^{3} \\
& =2.58596
\end{aligned}
$$

have been evaluated numerically. The dimensionality dependence of $\delta \lambda_{k}$ comes from the momentum integration

$$
\int \frac{\mathrm{d} \mathbf{p}}{(2 \pi)^{2}} \frac{(\mathbf{k} \cdot \mathbf{p})^{2}}{p^{6}}=\frac{S_{d} k^{2}}{(2 \pi)^{d} d} \int_{n^{\prime}}^{n} \mathrm{~d} p p^{d-5}
$$

where $S_{d}=2 \pi^{d / 2} / \Gamma(d / 2)$ is the surface area of the $d$-dimensional unit sphere. The upper cut-off $n \sim b^{-1}$ and the lower cut-off $n^{\prime} \sim N^{-1 / 2} b^{-1}$ are determined by the limits of validity of equation (11). For $d=4$ and $N$ large we therefore obtain for the diffusion coefficient (using the relation $D=\lambda_{0 k} / k^{2}$ )

$$
D=(\gamma / N)\left[1-2 c S_{d} \Delta /\left((2 \pi)^{d} b^{d} \ln N\right)\right]
$$

with $c=1.5872$. When $d>4$ the factor modifying $(\gamma / N)$ is independent of $N$, while for $d<4$ the pertur- 
bation expansion is in the variable $\left[\Delta N^{\varepsilon / 2}\right] / b^{d}$. Clearly four is the upper critical dimension for the dynamics. Were $\left(\Delta / b^{d}\right)$ to have a fixed point of order $\varepsilon=4-d$ in a renormalization group transformation, then it would be possible to exponentiate the logarithm in equation (14) and thereby determine the exponent governing the molecular weight dependence of the diffusion coefficient. The renormalization group procedure we have used is the "blobbing" transformation of Gabay and Garel [6]. This procedure has been extended to polymer dynamics by Al-Noaimi et al. [7].

The chain of $N$ beads $(N \rightarrow \infty)$ is divided into blobs of $g$ links; the blobs are then considered as new beads and the procedure is iterated. After the $(p+1)^{12}$ step the blobs are characterized to lowest order by a size $\left\langle L_{p+1}^{2}\right\rangle=g\left\langle L_{p}^{2}\right\rangle\left(\left\langle L_{1}^{2}\right\rangle=b^{2} d\right)$, excluded volume $V_{p+1}=g^{2} V_{p}$ and move in an effective random potential characterized by

$$
\Delta_{p+1}=g^{2} \Delta_{p}
$$

Since we are dealing with a quenched random system ln $Z$ must be averaged over $\varphi(\mathbf{R})$ rather than $Z$ itself, so we used the replica trick of writing

$$
\ln Z=\operatorname{Lim}\left(Z^{n}-1\right) / n
$$

as $n \rightarrow 0$ [4] and then averaged $Z^{n}$. It is useful to form the dimensionless quantities,

$$
\tilde{V}_{p}=\left(\frac{d}{2 \pi}\right)^{d / 2} \frac{V_{p}}{\left\langle L_{p}^{2}\right\rangle^{d / 2}} ; \quad \tilde{\Delta}_{p}=\left(\frac{d}{2 \pi}\right)^{d / 2} \frac{\Delta_{p}}{\left\langle L_{p}^{2}\right\rangle^{d / 2}} \text {. }
$$

one then readily derives, by conventional perturbation expansions [3] for the parameters of the "blobbed" effective replicated potential, the following approximate recursion relations, valid to first order in $\varepsilon$ (assuming $\tilde{V}_{p}, \tilde{\Delta}_{p} \sim \varepsilon$ ),

$$
\begin{aligned}
& \tilde{V}_{p+1}=g^{\varepsilon / 2} \tilde{V}_{p}\left[1-4 \tilde{V}_{p} \ln g+6 \tilde{\Delta}_{p} \ln g\right] \\
& \tilde{\Delta}_{p+1}=g^{\varepsilon / 2} \tilde{\Delta}_{p}\left[1-2 \tilde{V}_{p} \ln g+4 \tilde{\Delta}_{p} \ln g\right] \\
& D_{p+1}=g^{-1} D_{p}\left[1-c \tilde{\Delta}_{p} \ln g\right] .
\end{aligned}
$$

To analyse these recursion relations it is useful to go to the continuous limit, $p \delta=l, g=\exp \delta$ with $\delta \ll 1$, when setting $w=\tilde{V}-\tilde{\Delta}$ one has

$$
\begin{aligned}
& \frac{\mathrm{d} w}{\mathrm{~d} l}=w(\varepsilon / 2-4 w) \\
& \frac{\mathrm{d} \tilde{\Delta}}{\mathrm{d} l}=\tilde{\Delta}(\varepsilon / 2+2 \tilde{\Delta}-2 w) .
\end{aligned}
$$

In this form the recursion relations for $w, \tilde{\Delta}$ are a special case of those in reference [4]. Equation (15) has the stable fixed point $w^{*}=\varepsilon / 8$ but putting $w$ equal to this fixed point value in equation (16) one finds that $\widetilde{\Delta}(l)$ does not reach a fixed point as $l \rightarrow \infty$ if initially $\tilde{\Delta}>0$ (as must be supposed from the meaning of $\Delta$ cf. equation (5)). The stable fixed point at $\tilde{\Delta}^{*}=-\varepsilon / 8$, $w^{*}=\varepsilon / 8$ is unphysical by the same argument since it cannot be reached from the domain of initial values $w>0, \tilde{\Delta}>0$. It is a straightforward exercise to show that it is the quantity $w^{\prime}=V-\Delta$ which appears in all calculations of static quantities such as its radius of gyration. This can be done (for example) by examining the perturbation expansion in powers of $V$ and $\Delta$ to all orders [8]. Then the presence of a fixed point for $w$ implies that the random potential does not alter the usual exponents which describe the static properties of polymer chains. However, the absence of an accessible fixed point of order $\varepsilon$ for $\tilde{\Delta}(l)$ precludes a conventional exponent description for $D$.

What conclusions should be drawn from these calculations. We have shown that for $d>4$ the random potential merely modifies the friction coefficient $\gamma$ and reptation is not the mechanism of transport. For $d<4$ there is a radical alteration in the dynamics. The perturbation expansion for $d<4$ is of the form

$$
D=(\gamma / N) f\left(\Delta N^{\varepsilon / 2} / b^{d}\right) .
$$

The simplest possibility is that $f(x)$ varies as $x^{-2 / \varepsilon}$ as $x \rightarrow \infty$, so that $D \sim N^{-2}-$ the reptation result. The reptation model has no obvious connection with renormalization group considerations of behaviour on successive increasing scales of length and it might just be impossible to derive the reptation results from a renormalization group calculation. Another possibility is that the random potential localizes the eigenvectors of $A$ (other than the ground state) in the sense of Anderson [9] when $d<4$. This would imply that the diffusion coefficient was zero. Computer simulations [10] might reveal if this hypothesis is valid.

Acknowledgements. - G.C.M. wishes to thank the National Autonomous University of Mexico for a scholarship. We also gratefully acknowledge many useful discussions with Drs. G. Al-Noaimi, A. J. Bray, D. Elderfield and C. A. Wilson.

\section{References}

[1] de Gennes, P. G., Scaling Concepts in Polymer Physics (Cornell U.P., Ithaca) 1979, Chapter 8.

[2] Rouse, P. E., J. Chem. Phys. 21 (1953) 1272.

[3] Yamakawa, H., Modern Theory of Polymer Solutions (Harper and Row, New York) 1971, Chapter 6.

[4] Grinstein, G. and Luther, A., Phys. Rev. B 13 (1976) 1329.

[5] de Gennes, P. G., Physics 3 (1967) 37.
[6] Gabay, M. and Garel, T., J. Physique Lett. 39 (1978) L-123. [7] Al-Noaimi, G. F., Martinez-Mekler, G. C. and Wilson, C. A., J. Physique Lett. 39 (1978) L-373.

[8] BraY, A. J. and Moore, M. A., never to be published.

[9] Anderson, P. W., Phys. Rev. 109 (1958) 1492.

[10] BAUMGÄRTER, A. and BINDER, K., preprint. 